\title{
Revisión
}

\section{Estado actual de la vacuna conjugada contra Streptococcus pneumoniae}

\author{
Hernán Sierra- Fernandez', Malka Schultz- Faingezicht', Carolina Soley-Gutiérrez', \\ Silvia Guevara- Jiménez', Adriano Arguedas- Mohs ${ }^{1,2}$
}

\section{Resumen}

Las infecciones por Streptococcus pneumoniae son frecuentes en la población pediátrica especialmente en los niños menores de 2 años. El S. pneumoniae puede producir infecciones invasoras con una alta tasa de mortalidad y morbilidad como lo son las meningitis bacterianas, la neumonía y bacteremias siendo a la vez el agente que con mayor frecuencia se detecta en el oído medio de niños con otitis media. En la actualidad existe una vacuna conjugada contra esta bacteria que protege contra los siete serotipos de S. pneumoniae más frecuentes en el mundo y que a su vez son los mismos serotipos que presentan una mayor incidencia de resistencia a los antibioticos de uso frecuente. La vacuna no solo protege contra este tipo de infecciones sino que se ha demostrado que disminuye la colonización nasofaringea de los niños que han recibido la vacuna produciendo a su vez, una reducción en el numero de infecciones, por esta bacteria, en poblaciones de personas mayores de 5 años, incluyendo adultos y personas mayores a los 65 años (efecto rebaño). Con base en los serotipos aislados en niños costarricenses con otitis media, se puede calcular que la cobertura de esta vacuna en Costa Rica sería de aproximadamente un $74 \%$ e incluyendo mayoritariamente, los serotipos que presentan resistencia antimicrobriana más frecuentemente.

Palabras clave: S. pneumoniae, enfermedad invasora, vacuna conjugada.

Keywords: S. pneumoniae, vaccine, serotype.

Recibido: 1 de noviembre de 2005

Aceptado: 17 de enero de 2006

Instituto de Atención Pediátrica

2 Universidad de Ciencias Médicas

\section{Correspondencia:}

Dr. Adriano Arguedas Mohs. Pediatra Infectólogo, Director Instituto de Atención Pediátrica. Apartado Postal \#607-I I 50 La Uruca, San José.

ISSN 0001-6002/2006/48/2/66-71 Acta Médica Costarricense, @2006 Colegio de Médicos y Cirujanos
El Streptococcus pneumoniae es una bacteria que habitualmente coloniza el tracto respiratorio en los seres humanos. Es la principal causa de infección bacteriana de las vías respiratorias y su pico de incidencia es en niños menores de dos años de edad y en personas mayores de 65 años $^{1,2}$. En la población pediátrica, el $S$. pneumoniae es el principal agente etiológico de infecciones como: otitis media, sinusitis y otras más como: neumonía, bacteremia y meningitis ${ }^{2,3}$.

Los casos de neumonía y meningitis causados por este germen afectan anualmente a más de un millón de niños alrededor del mundo, y producen serias secuelas o muerte en un alto porcentaje de estos ${ }^{4}$. En las últimas décadas el tratamiento de estas infecciones se ha complicado debido a un rápido y progresivo aumento en el patrón de resistencia de este germen a la penicilina y a otros antibióticos; este hecho ha generado preocupación en las autoridades de salud alrededor del mundo las cuales han sugerido la aplicación de medidas preventivas contra las infecciones por $S$. pneumoniae ${ }^{4,5}$.

En el pasado, la vacuna conjugada contra Haemophilus influenzae tipo B demostró ser de gran utilidad, ya que redujo la colonización y diseminación de esta bacteria en niños y 
disminuyó en un $98 \%$ la incidencia de infecciones invasoras por Haemophilus influenzae tipo $\mathrm{B}^{6,7}$. El éxito observado con esta vacuna impulsó la creación de la primera vacuna conjugada contra Streptococcus pneumoniae. A diferencia de la vacuna-no conjugada ya existente, que contiene 23 polisacáridos (Pneumo23 § o Pneumovax $\left({ }^{\circledR}\right)$ ), la nueva vacuna conjugada, induce memoria inmunológica en niños menores de dos años, disminuyendo en esta población, la colonización por $S$. pneumoniae en la nasofaringe y la orofaringe.

Basada en los resultados de diversos estudios con la vacuna conjugada contra S. pneumoniae, en 2001 la Administración de Drogas y Alimentos (FDA) de los Estados Unidos recomendó su uso en niños a partir de los 2 meses de edad ${ }^{8}$. En Costa Rica esta vacuna se encuentra, en el sector privado, desde principios de 2004.

Este artículo hace una revisión de los estudios realizados antes y después de la aprobación de la vacuna y analiza el efecto que ha tenido sobre la enfermedad neumocóccica en el mundo y el impacto que su uso podría tener en Costa Rica.

\section{Epidemiología a nivel mundial}

La Organización Mundial de la Salud reporta que el Streptococcus pneumoniae produce de 800,000 a 1 millón de casos de neumonía o de meningitis por año en la población pediátrica a nivel mundial. En países en vías de desarrollo, un alto porcentaje (60\%-90\%) de los casos de neumonía por $S$. pneumoniae producen la muerte y entre el 40 y el $75 \%$ de los casos de meningitis generan muerte o discapacidad ${ }^{4}$.

En Estados Unidos, en 1997, el S. pneumoniae fue el responsable de 790 casos de meningitis, 15,000 bacteremias, 77,000 neumonías, 7 millones de casos de otitis media, y de la colocación de aproximadamente 149,000 tubos timpánicos ${ }^{8}$.

En Costa Rica, datos retrospectivos obtenidos del Hospital Nacional de Niños durante el período 1995-2001, indican que la incidencia de enfermedad neumocóccica invasora fue de 19.3 casos por año (42\% meningitis, $27 \%$ neumonía y $22 \%$ bacteremia), con una mortalidad del $14 \%$ y complicaciones tales como falla respiratoria, muerte o shock séptico en un $49 \%$ de los casos ${ }^{9}$.

Los costos anuales estimados en el tratamiento de episodios de otitis media por neumococo son de más de 138 millones de dólares en Finlandia (población 5 millones) ${ }^{10,11}$ y hasta de 5 billones de dólares en Estados Unidos ${ }^{12-14}$.

\section{Microbiología del Streptococcus pneumoniae}

El Streptococcus pneumoniae es un diplococo gram positivo, encapsulado que produce una hemólisis alfa en agar sangre y es sensible a optoquina ${ }^{15}$. $\mathrm{Su}$ reservorio exclusivo es la nasofaringe humana ${ }^{5}$. La afinidad por la mucosa respiratoria está dada por una proteína fijadora de colina, la cual es altamente específica y afín a las células epiteliales nasofaríngeas ${ }^{16}$. El neumococo vive en la nasofaringe por períodos prolongados (2.5 a 4.5 meses), en donde puede permanecer latente o invadir produciendo infección ${ }^{17}$. La severidad de la infección depende del serotipo de neumococo que esté actuando, ya que la cantidad de factores de virulencia varía de un serotipo a otro ${ }^{18}$. Uno de estos factores de virulencia que ha adquirido importancia en los últimos años es su proteína fijadora de penicilina, la cual es un factor de resistencia a la penicilina.

\section{La cápsula de polisacáridos}

La cápsula del Streptococcus pneumoniae es sin duda el factor de virulencia más importante, ya que confiere protección contra la respuesta inmunológica ${ }^{19}$. Se compone de una densa capa de polisacáridos, entre los cuales se encuentran estructuras altamente repetitivas conocidas como epítopos. Estos son determinables por pruebas serológicas y hoy en día se conocen más de 90 serotipos, de los cuales un pequeño porcentaje tiene mayor importancia clínica en el ser humano.

En los Estados Unidos, los serotipos 4, 6B, 9V, 14, 18C, $19 \mathrm{~F}$ y $23 \mathrm{~F}$ son los responsables del $80 \%$ de las infecciones en el hombre y del $80 \%$ de los casos de resistencia bacteriana ${ }^{20}$.

La distribución de los serotipos de S. pneumoniae que causan otitis media en niños costarricenses ha sido analizada en cepas aisladas del oído medio de estos niños, durante el período 1999 - $2003^{21,22}$. Interesantemente, luego de un pico muy importante de varios clones del serotipo 19F (75\%) durante 1999 - 2001, su incidencia disminuyó considerablemente 2002-2003 (26\%) y la distribución de serotipos, en este último período, indicó que además del serotipo $19 \mathrm{~F}$ otros tipos relevantes son: 6B (15\%), 9V (9\%), 16F (9\%), 14 (6\%), 23F (6\%), 3 (6\%) y el 6A (6\%). Similar a estudios publicados previamente por otros centros, se logró detectar como los serotipos que se encuentran dentro de la nueva vacuna conjugada heptavalente presentan una tendencia a poseer un porcentaje de resistencia a penicilina y trimetroprim-sulfametoxazole, más alta que la observada en los serotipos que no están dentro de la vacuna conjugada ${ }^{21,22}$.

\section{Colonización con Streptococcus pneumoniae}

La mayoría de los niños son colonizados en la nasofaringe y en la orofaringe por el Streptococcus pneumoniae durante los primeros meses de vida ${ }^{24}$. Al mes de edad el $3.6 \%$ de los niños están colonizados con S. pneumoniae y este porcentaje aumenta conforme avanza la edad. Al año de edad, el 19\% de los niños están colonizados por esta 
bacteria y un $95 \%$ habrá sido colonizado al menos una vez durante los primeros 12 meses de vida ${ }^{25}$. Durante este período, la transmisión del S. pneumoniae se da directamente de persona, a persona por pequeñas gotas de saliva o fomites, y ocurre en mayor grado cuando hay condiciones de hacinamiento ${ }^{17,24}$.

La colonización es necesaria para que se produzca la infección, sin embargo, solo un $15 \%$ de las personas colonizadas desarrollarán un proceso infeccioso ${ }^{20}$. Los factores predisponentes para que se presente una infección son: edad inferior a los dos años, asistencia a guarderías, infección viral de las vías respiratorias y exposición al humo del cigarro $^{5,26}$.

Diversos estudios han demostrado cómo la población pediátrica tiene un mayor inóculo de bacterias colonizantes en la nasofaringe y la orofaringe. Además, esta colonización tiende a ser por períodos prolongados ${ }^{5}$. Como consecuencia de estos dos factores, los niños tienen una mayor posibilidad de estar colonizados por cepas resistentes a antibióticos convencionales ${ }^{18}$.

\section{Respuesta inmunológica: la respuesta T-independiente}

En los seres humanos, la respuesta inmunológica ante una infección bacteriana está mediada por los linfocitos $\mathrm{T}^{26}$, que procesan los antígenos de la superficie de la bacteria, dando como resultado la producción de altas cantidades de anticuerpos tipo $\operatorname{IgG}$ específicos contra dicho antígeno, lo que se conoce como memoria inmunológica. Esto permite que, ante un nuevo estímulo inmunológico, la opsonización $\mathrm{y}$ fagocitosis bacteriana se den en forma rápida y efectiva ${ }^{27}$.

La gruesa capa de polisacáridos presentes en las bacterias encapsuladas no es fácilmente reconocida por las células presentadoras de antígeno, y así, evade la respuesta mediada por los linfocitos T. Debido a esto, el sistema inmunológico queda sujeto a la respuesta de los linfocitos $\mathrm{B}$, los cuales deberán producir anticuerpos inespecíficos tipo $\operatorname{IgM}$.

En condiciones normales, los adultos producen cantidades suficientes de anticuerpos IgM en un tiempo adecuado para evitar una infección grave. Este proceso es muy distinto en niños menores de 2 años, en quienes los linfocitos $\mathrm{B}$ no producen cantidades suficientes de anticuerpos y por lo tanto a esta edad son más frecuentes las infecciones por Streptococcus pneumoniae ${ }^{27,28}$. Esta respuesta inmunológica T-independiente que genera el Streptococcus pneumoniae, representa la base de su fisiopatología y del mecanismo de acción de las nuevas vacunas conjugadas. Estas vacunas son conjugados proteína-polisacárido, en los cuales la parte proteica induce una respuesta T-dependiente con niveles altos de $\operatorname{IgG}$ específicos para la porción de polisacárido $^{29}$.

\section{Vacuna no conjugada}

La vacuna no conjugada de polisacáridos capsulares del Streptococcus pneumoniae protege contra los 23 serotipos más frecuentes en el humano. Sin embargo, debido a la naturaleza T-independiente del polisacárido capsular, esta vacuna no induce respuesta en niños menores de 2 años y no ofrece ningún efecto sobre la colonización en las vías respiratorias ${ }^{30}$. Así, la vacuna no conjugada de polisacáridos está recomendada únicamente bajo ciertas circunstancias, en adultos y niños mayores de dos años, en quienes sí genera protección inmunológica ${ }^{31}$.

\section{Vacuna conjugada}

Durante la década de los 90 comenzó la elaboración de vacunas conjugadas contra Streptococcus pneumoniae. Las nuevas vacunas incluyen desde 7 hasta 11 de los serotipos más frecuentes causantes de enfermedad neumocóccica en el hombre ${ }^{32,33}$. Sin embargo, solo la vacuna heptavalente ha sido aprobada para su uso en niños menores de 2 años; ésta es un conjugado de los 7 serotipos más frecuentes $(4,6 \mathrm{~B}$, 9V, 14, 18C, 19F y 23F) unidos a la proteína CRM197, un mutante no tóxico de la toxina diftérica ${ }^{34}$.

\section{Efectividad de la vacuna}

Tres años antes de la aprobación de la vacuna por parte de la FDA, en 2000, Black y colaboradores estudiaron la inmunogenicidad, reactogenicidad y la eficacia clínica de la vacuna conjugada contra infecciones invasoras por $S$. pneumoniae, en niños menores de dos años ${ }^{35}$. Este fue un estudio de cohorte, a doble ciego, en el que cual se randomizaron 37,000 niños, quienes recibieron 4 dosis de la vacuna o placebo en un esquema en el que la vacuna o el placebo se aplicaron a los 2, 4, 6 y 12-15 meses de edad. Los resultados demostraron un alto nivel de eficacia. En niños que recibieron las 4 dosis, se observó una efectividad del $97 \%$ en la reducción de casos de enfermedad invasora, causados por los serotipos incluidos en la vacuna y una reducción del $89 \%$ en el número de infecciones globales causadas por $S$. pneumoniae, incluyendo aquellas producidas por serotipos no incluidos en la vacuna. Así mismo, se observó que aún en niños que recibieron 3 dosis de la vacuna hubo una reducción del $86 \%$ en el número de casos de enfermedad neumocóccica invasora. Además de analizar el impacto de la vacuna en la reducción de enfermedad sistémica neumocóccica, se estudió el impacto de la vacuna sobre la incidencia de casos clínicos de otitis media, observándose una reducción del $22 \%$ en el número de casos de otitis media recurrente y de un $20 \%$ en la colocación de tubos timpánicos.

Black y colaboradores también analizaron el efecto de la vacuna durante los 3 años posteriores a su introducción en el esquema básico de inmunización ${ }^{36}$. Los resultados coincidieron con el estudio clínico previo, documentando una reducción del $90 \%$ del total de los casos de enfermedad 
neumocóccica invasora en niños menores de 2 años de edad. La diferencia entre los resultados de la efectividad de la vacuna en este estudio (90\%) y los porcentajes de cobertura calculada para la vacuna $(80 \%)$, se explica por una reacción cruzada que ocurre con algunos serotipos no incluidos en la vacuna.

Los cambios en la incidencia de otitis media secundarios a la vacuna fueron estudiados tambien por Eskola et al. en Finlandia ${ }^{10}$. El estudio demostró una reducción del $57 \%$ en los episodios de otitis media causados por los serotipos de $S$. pneumoniae incluidos en la vacuna, una disminución del $34 \%$ en los casos de otitis media por S. pneumoniae en general y una reducción del $6 \%$ de casos de otitis media aguda. Similar a lo observado por el grupo de Kaiser Permanente ${ }^{36}$, los estudios de seguimiento han expuesto un impacto positivo en niños vacunados, en quienes se ha establecido una reducción del $39 \%$ en la colocación de tubos timpánicos ${ }^{37}$.

Los efectos adversos observados hasta el momento con la vacuna heptavalente han tenido incidencias similares a los del resto de las vacunas. Las reacciones más comunes son locales en el sitio de la inyección (enrojecimiento, inflamación y dolor) y el $100 \%$ resolvieron antes de 14 días. En los estudios clínicos, fiebre alta $\left(>39^{\circ} \mathrm{C}\right)$ ocurrió con una mayor frecuencia en la vacuna heptavalente que en las otras vacunas, sin embargo, su duración fue autolimitada y no produjo secuelas en ningún paciente ${ }^{35}$.

\section{Ventajas de la disminución en la colonización nasofaríngea}

Las vacunas conjugadas disminuyen la colonización nasofaríngea en niños menores de dos años de edad ${ }^{34,38}$. Estudios randomizados han demostrado reducciones significativas en la colonización con serotipos incluidos en la vacuna tres meses después de la primer dosis de la vacuna conjugada, efecto que no se observa con la vacuna no conjugada ${ }^{35}$. Al disminuir la colonización nasofaríngea de pacientes vacunados, la vacuna conjugada disminuye la transmisión a los familiares de estos niños. Este fenómeno denominado "inmunidad de rebaño" se detectó en un estudio, en el cual se demostró una reducción en la incidencia de enfermedad neumocóccica invasora del $32 \%$ en adultos de 20 a 39 años y de un $18 \%$ en personas mayores de 65 años ${ }^{34}$.

\section{Reducción en la resistencia a antibióticos}

Los siete serotipos incluidos en la vacuna heptavalente comprenden entre el $80 \%$ y el $90 \%$ de los serotipos resistentes a penicilina, por lo tanto una disminución en la colonización nasofaríngea por estos serotipos, tiene un efecto directo sobre la incidencia de serotipos resistentes a antibióticos ${ }^{39}$. Datos analizados en cepas de $S$. pneumoniae aisladas del oído medio de niños costarricenses, demuestran que en esta población también se observa este fenómeno de mayor resistencia antibiótica en los serotipos incluidos en la vacuna conjugada ${ }^{21}$.

En el estudio de Black y colaboradores realizado en California, se observaron cambios significativos en la incidencia de cepas resistentes a penicilina, ya que en 1999, previo a la introducción de la vacuna en los Estados Unidos, la resistencia a penicilina era de un $14 \%$ y tres años después de la introducción de la vacuna, esta resistencia se redujo a un 5\%; además, en este mismo estudio se observó una disminución en el porcentaje de resistencia a la eritromicina y a la tetraciclina ${ }^{36}$.

La disminución de la resistencia antimicrobiana del S. pneumoniae ha llevado a una reducción en el número de fallas terapéuticas y consecuentemente, a una disminución en el uso de antibióticos. En un estudio publicado recientemente se demostró que, en promedio, luego de la primera dosis, la vacuna conjugada previno un total de 35 prescripciones de antibióticos por cada 100 niños vacunados ${ }^{39}$.

\section{El fenómeno de reemplazo}

La reducción en la colonización por serotipos incluidos en la vacuna se ha visto asociada a un reemplazo por serotipos no incluidos en la vacuna ${ }^{10,20}$. Sin embargo, este fenómeno se ha observado solamente en el caso de otitis media aguda, en el que se ha notado un aumento del $30 \%$ en la incidencia de serotipos no incluidos en la vacuna ${ }^{10}$. A diferencia de esto, en los casos de enfermedad invasora no se han podido demostrar aumentos estadísticamente significativos en la incidencia de serotipos no incluidos en la vacuna ${ }^{34}$.

\section{Estado actual de la vacuna conjugada en Costa Rica}

En vista de la morbilidad y mortalidad de enfermedades producidas por $S$. pneumoniae en Costa Rica y debido a que la vacuna conjugada fue introducida en el sector privado a principios de 2004, es importante conocer cuál podría ser el impacto que la aplicación de esta vacuna conjugada tendría en Costa Rica 9, 21, 22, 40,41.

En el país, se ha analizado la cuáles son los serotipos de S. pneumoniae más frecuentes en el oído medio de niños costarricenses con otitis media (Cuadro 1) ${ }^{21}$. En el primer análisis efectuado en cepas aisladas entre los años 19992001 se detectó que el serotipo 19F era aislado en el 79\% de estos pacientes ${ }^{22}$. Datos obtenidos en una población similar, durante el período 2002-2003, demostraron que a pesar de que el serotipo $19 \mathrm{~F}$ sigue siendo el más frecuente, su incidencia se redujo a un $26 \%$, mientras durante ese período de tiempo, la incidencia de otros serotipos aumentó, permitiendo calcular que la protección esperada con la vacuna heptavalente en Costa Rica sería de un $74 \%$.

Recientemente, estudios de genética molecular llevados a cabo con el serotipo 19F aislado del oído medio de pacientes en Costa Rica, demostraron una relación muy 
estrecha a nivel de ADN, con un serotipo 15B/C aislado en niños israelíes ${ }^{42}$. Este hallazgo ha sido interpretado de manera que los dos serotipos, el 19F aislado en Costa Rica y el $15 \mathrm{~B} / \mathrm{C}$ aislado en Israel, fueron en un principio el mismo serotipo (probablemente 19F) y que la cepa aislada en Israel cambió su polisacárido externo para transformarse de un serotipo incluido en la vacuna (19F) a otro no incluido en la vacuna $(15 \mathrm{~B} / \mathrm{C})$. Este fenómeno, no reportado previamente, se conoce como "cambio capsular" (capsular switch) y en principio podría ser un mecanismo de resistencia a la vacuna.

\section{Conclusión}

La vacuna conjugada de $S$. pneumoniae ha demostrado ser segura y eficaz en prevenir infecciones invasoras y la otitis media por $S$. pneumoniae en la población pediátrica. Al incluir los serotipos más frecuentes causantes de infección en el hombre, la vacuna ha logrado reducciones dramáticas en la incidencia de enfermedad pneumocóccica invasora en niños menores de dos años y al reducir la colonización nasofaringea en los niños ha disminuido la transmisión niño-adulto, reduciendo también las infecciones invasoras por este germen en adultos. Además, ha logrado disminuir el número de cepas con resistencia a diversos antibióticos $\mathrm{y}$, consecuentemente, el número de casos con falla terapéutica a antibióticos convencionales. Con base en la incidencia de serotipos de $S$. pneumoniae en Costa Rica, se puede predecir una cobertura nacional del $74 \%$, lo cual significaría una importante reducción de la enfermedad sistémica por $S$. pneumoniae.

\section{Agradecimiento}

A la Sra. María Virginia Urcuyo Fournier y a la Master Sandra Ramírez, por su ayuda en la edición del manuscrito.

\section{Abstract}

The heptavalent $S$. pneumoniae conjugate vaccine has shown to be safe and effective in preventing pediatric invasive infections and otitis media caused by $S$. pneumoniae. The routine use of these vaccines has dramatically reduced the incidence of invasive pneumococcal diseases in children younger than 2 years old and because of a reduction in colonization of the children's nasopharynx, the transmission from child to adult has been reduced and the number of secondary $S$. pneumoniae infections in adults. Another benefit of the routine use of this vaccine has been the reduction of vaccine-type antimicrobial resistant strains. Based on the $S$. pneumoniae serotype distribution in Costa Rica, the predicted vaccine protection is calculated in $74 \%$.

\section{Referencias}

1. Preventing pneumococcal disease among infants and young children: recommendations of the Advisory Committee on Immunization Practices (ACIP) MMWR 2000; 49:1-38.

2. MMWR CDC Prevention of pneumococcal disease: recommendations of the Advisory Committee on Immunization Practices (ACIP). 1997; 46:1-24.

3. Peter G, Klein JO. Streptococcus pneumoniae. En: Long SS, Pickering LK, Prober CG, eds. Principles and practice of pediatric infectious diseases. 2nd ed. Philadelphia: Churchill Livingstone. 2003:739-46

4. World Health Organization. Pneumococcal vaccine.Wkly Epidemiol Report 2003; 14:110-9.

5. Dagan R, Relamed M, Piglansky L, Greenberg D, Abramson O, Mendelman PM, et al. Reduction of nasopharyngeal carriage of pneumococci during the second year of life by a heptavalent conjugate pneumococcal vaccine. J Infect Dis. 1996; 174:1271-78.

6. Shinefield HR, Black SB. Postlicensure surveillance for Haemophilus influenzae type $\mathrm{b}$ invasive disease alter use of Haemophilus influenzae type b oligosaccharide CRM197 conjugate vaccine in the large defined United Status population: a four year eight month follow up. Pediatr Infect Dis J. 1995; 14:978-981.

7. CDC. Progress toward elimination of Haemophilus influenzae type $b$ among infants and children: United States, 1987-1993. MMWR 1994; 43:144-8.

8. Centers for Disease Control and Prevention. Prevention of pneumococcal disease: recommendation of the Advisory Committee on Immunization Practices (ACIP), MMWR. 1997; 46:1-24.

9. Ulloa R, Avila M, Herrera M, Herrera J, Arguedas A. Invasive pneumococcal disease in Costa Rican children: a seven year survey. Pediatr Infect Dis J. 2003; 22:1069-74.

10. Eskola J, Kilpi T, Palmu A, Jokinen J, Haapakoski J, Herva E, et al. Efficacy of a Pneumococcal conjugate vaccine against acute otitis media. N Engl J Med. 2001; 344:403-409.

11. Niemelä M, Uhari M, Möttönen M, Pokka T. Cost arising from otitis media. Acta Paediatr. 1999; 88:553-556.

12. Gates GA. Cost-effectiveness considerations in otitis media treatment. Otolaryngol Head Neck Surg. 1996; 14:525-530.

13. Kaplan B, Wandstrat TL, Cunningham JR. Overall cost in the treatment of otitis media. Pediatr Infect Dis J. 1997; 16:S9-S11.

14. Bondy J, Berman S, Glazner J, Lezotte D. Direct expenditures related to otitis media diagnosis: extrapolations from a pediatric Medicaid cohort. Pediatrics. 2000; 105:1323-1323.

15. National Committee for Clinical and Laboratory Standards: twelfth informational supplement. NCCLS document. M100-S12 (ISBN 156238-454-6). NCCLS, 940 West Valley Road, Suite 1400, Wayne, Pennsylvania 12807-1898, USA 2002.

16. Hava DL, Le Mieux J. From nose to lung: the regulation behind Streptococcus pneumoniae virulence factors. Molec Microbiol. 2003; 50:1103-10.

17. Ghaffar F, Friedland I, Mccraken G. Dynamics of nasopharyngeal colonization by S.pneumoniae. Pediatr Infect Dis J. 1999; 18: 638-46. 


\section{Streptococcus pneumoniae / Sierra $\mathrm{H}$ et al}

18. Brueggemann AB, Peto TE, Crook DW, Butler JC, Kristinsson K, Spratt B. Temporal and geographic stability of the serogroup- specific invasive disease potential of Streptococcus pneumoniae in children. J Infect Dis. 2004; 190:1203-11.

19. Fernebro J, Andersson I, Sublett J, Morfeldt E, Novak R, Tuomanen E, et al. Capsular expression in Streptococcus pneumoniae negatively affects spontaneous and antibiotic-induced lysis and contributes to antibiotic tolerance. J Infect Dis. 2004; 189:328-38. Epub 2003 Dec 30.

20. American Academy of Pediatrics. Pneumococcal Infections En: Pickering LK, ed. Red book: 2003 Report of the Committee on Infectious Diseases. 26th ed. Elk Grove Village, IL: American Academy of Pediatrics; 2003:496-500.

21. Arguedas A, Dagan R, Guevara S, Porat N, Soley C, Pérez A, Brilla R. Middle ear fluid Streptococcus pneumoniae serotype distribution in Costa Rican children with otitis media. Pediatr Infect Dis J. 2005; 24:631-634

22. Arguedas A, Dagan R, Soley C, Loaiza C, Knudsen K, Porat N, Perez A, Brilla E, Herrera ML. Microbiology of otitis media in Costa Rican children, 1999 through 2001. Pediatr Infect Dis J. 2003; 22:1063-8.

23. Dagan R. Vacunas neumocóccicas conjugadas para prevenir la portación y propagación de neumococos resistentes a los antibióticos. Arch. argent pediatr. 2002:100:19-24.

24. Vives M, García ME, Saenz P, Mora M, Mata L, Sabharwal H, et al. Nasopharyngeal colonization in Costa Rican children during the first year of life. Pediatr Infect Dis J. 1997; 16:852-58.

25. García JA, Fresnadillo MJ. Dynamics of nasopharyngeal colonization by potential respiratory pathogens. J Antimicrob Chemother. 2002; 50:59-73.

26. Fattom A, Lue C, Szu SC, Mestecky J, et al. Serum antibody response in adult volunteers elicited by injection of Streptococcus pneumoniae type $12 \mathrm{~F}$ polysaccharide alone or conjugated to diphtheria toxoid. Infect Immun. 1990; 58:2309-12.

27. Nieminen T, Kayhty H, Leroy O, Eskola J. Pneumococcal conjugate vaccination in toddlers: mucosal antibody response measured as circulating antibody-secreting cells and as salivary antibodies. Pediatr Infect Dis J. 1999; 18:764-72.

28. Adderson EE. Antibody repertoires in infants and adults: effects of T-independent and T-dependent immunizations. Springer Semin Immunopathol. 2001; 23:387-403.

29. Perez-Melgosa M, Ochs HD, Linsley PS, Laman JD, van Meurs M, Flavell R, et al. Carrier-mediated enhancement of cognate $\mathrm{T}$ cell help: the basis for enhanced immunogenicity of meningococcal outer membrane protein polysaccharide conjugate vaccine. Eur J Immunol. 2001; 31:2373-81.

30. Wasserman RL, Sorensen R. Evaluating children with respiratory tract infections: the role of immunization with bacterial polysaccharide vaccine. Pediatr Infect Dis J. 1999; 18:157-163.
31. Huo Z, Spencer O, Miles J, Johnson J, Holliman R, Sheldon J, et al. Antibody response to pneumolysin and to pneumococcal capsular polysaccharide in healthy individuals and Streptococcus pneumoniae infected patients. Vaccine. 2004; 22:1157-61.

32. Obaro SK, Adegbola RA, Chang I, Banya WA, Jaffar S, Mcadam K, et al. Safety and immunogenicity of a nonavalent pneumococcal vaccine conjugated to CRM 197 administered simultaneously but in a separate syringe with diphtheria, tetanus and pertussis vaccines in Gambian infants. Pediatr Infect Dis J. 2000; 19: 463-469.

33. Dagan R, Kaythty H, Wuorimaa T, Yaich M, Bailleux F, Zamir O, et al. Tolerability and immunogenicity of an eleven valent mixed carrier Streptococcus pneumoniae capsular polysaccharide-diphtheria toxoid or tetanus protein conjugate vaccine in Finnish and Israeli infants. Pediatr Infect Dis J. 2004; 23:91-98.

34. Whitney C, Farley M, Hadler J, Harrison L, Bennett NM, Lynfield R, et al. Decline in invasive pneumococcal disease after the introduction of protein-polysaccharide conjugate vaccine. N Engl J Med. 2003; 348:1737-46

35. Black S, Shinefield H, Fireman B, Lewis E, Ray P, Hansen J, et al. Efficacy, safety and immunogenicity of heptavalent pneumococcal conjugate vaccine in children. Pediatr Infect Dis J. 2000; 19: 187195.

36. Black S, Shinefield H, Baxter R, Austrian R, Bracken L, Hansen J, et al. Postlicensure surveillance for pneumococcal invasive disease after use of heptavalent pneumococcal conjugate vaccine in Northern California Kaiser Permanente. Pediatr Infect Dis J. 2004; 23: 485489.

37. Palmu AI, Verho J, Jokinen J, Karma P, Kilpi T. The seven valent pneumococcal conjugate vaccine reduces tympanostomy tube placemente in children. Pediatr Infect Dis J. 2004; 23:732-738.

38. Hausdorff W, Yothers G, Dagan R, Kilpi T, Pelton S, Cohen R, et al. Multinacional study of pneumococcal serotypes causing acute otitis media in children. Pediatr Infect Dis J. 2002; 21:1008-16.

39. Dagan R, Givon-Lavi N, Shkolnik L, Yagupsky P, Fraser D. Acute otitis media caused by antibiotic-resistant Streptococcus pneumoniae in southern Israel: implication for immunizing with conjugate vaccines. J Infect Dis. 2000; 181:1322-9.

40. Fireman B, Black SB, Shinefield HR, Lee J, Lewis E, Ray P. Impact of the pneumococcal conjugate vaccine on otitis media. Pediatr Infect Dis J. 2003; 22:10-6.

41. Arguedas A, Loaiza C, Pérez A, Vargas F, Herrera M, Rodríguez G, et al. Microbiology of otitis media in Costa Rican children. Pediatr Infect Dis J. 1998; 17:680-9.

42. Porat N, Arguedas A, Spratt B, Trefler R, Brilla E, Loaiza C, et al. Emergence of penicillin nonsusceptible Streptococcus pneumoniae clones of non-vaccine serotypes: Serotype $11 \mathrm{~A}$ and $15 \mathrm{~B} / \mathrm{C}$ variants of resistant clones of serotypes included in the pneumococcal conjugate vaccines. J Infect Dis. 2004; 190:2154-2161. 